\title{
UNIQUENESS OF THE UNIFORM NORM WITH AN APPLICATION TO TOPOLOGICAL ALGEBRAS
}

\author{
S. J. BHATT AND D. J. KARIA
}

(Communicated by Palle E. T. Jorgensen)

\begin{abstract}
Any square-preserving linear seminorm on a unital commutative algebra is submultiplicative; and the uniform norm on a uniform Banach algebra is the only uniform $Q$-algebra norm on it. This is proved and is used to show that (i) uniform norm on a regular uniform Banach algebra is unique among all uniform (not necessarily complete) norms and (ii) a complete uniform topological algebra that is a $Q$-algebra is a uniform Banach algebra. Relevant examples, showing that the respective assumptions regarding regularity, $Q$-algebra norm, and uniform property of topology cannot be omitted, have been discussed.
\end{abstract}

\section{INTRODUCTION}

We prove the following

Theorem 1. (i) Any linear norm with square property on a commutative algebra is an algebra norm.

(ii) Let $(A,\|\cdot\|)$ be a uniform Banach algebra. Let $|\cdot|$ be a linear norm with square property on $A$ such that the set $A^{-1}$ of invertible elements forms an open set in $(A,|\cdot|)$. Then $\|\cdot\|=|\cdot|$.

Corollary. Let $(A,\|\cdot\|)$ be a regular uniform Banach algebra. Let $|\cdot|$ be any norm on $A$ such that $(A,|\cdot|)$ is a normed algebra. Then $\|\cdot\| \leq|\cdot|$. Additionally, if $|\cdot|$ is a uniform norm then $\|\cdot\|=|\cdot|$.

Theorem 1 is used to prove the following

Theorem 2. Let $A$ be a complete uniform topological algebra that is a $Q$-algebra. Then the topology of $A$ is normable and $A$ is a uniform Banach algebra.

After briefly discussing the preliminaries in $\S 1$, the proofs are presented in $\S 2$. In $\S 3$ we discuss some relevant remarks and examples showing that various assumptions of the above results cannot be omitted.

Received by the editors December 14, 1990 and, in revised form, March 25, 1991; presented at the 56th Annual Conference of the Indian Mathematical Society on December 30, 1990.

1991 Mathematics Subject Classification. Primary 46J05, 46J10, 46J05.

Key words and phrases. Uniform Banach algebra, regular Banach algebra, topological algebra, $Q$-algebra.

The second author was a Junior Research Fellow of the University Grants Commission, India, at the time of the preparation of this paper. 


\section{Preliminaries}

By an algebra we mean a linear associative algebra $A$ with complex scalars and having identity 1 . A norm $|\cdot|$ is a linear norm if $(A,|\cdot|)$ is a normed linear space. Such a norm has the square property (respectively, is an algebra norm) if $\left|x^{2}\right|=|x|^{2}$ for all $x$ (respectively, if $|x y| \leq|x||y|$ for all $x, y$ ). A uniform (semi)norm is an algebra (semi)norm with square property. We do not assume, in either of these cases $(A,|\cdot|)$ to be complete. A uniform Banach algebra [5] is a Banach algebra $(A,\|\cdot\|)$ with uniform norm $\|\cdot\|$. Gelfand theory describes $A$ as a closed subalgebra of the supnorm Banach algebra $C(X)$ (= all continuous functions on a compact Hausdorff space $X$ ) separating points of $X$ and containing constants. For regular Banach algebras, we refer to [5, Chapter 7].

A topological algebra $(A, \tau)$ is a Hausdorff topological vector space $A$ (having topology $\tau$ ) that is an algebra with separately continuous multiplication. It is a complete uniform topological algebra if it is complete and $\tau$ is determined by a separating family $P$ of uniform seminorms. One can replace $P$ by the family $S(A)$ of all continuous uniform seminorms. For each $p \in S(A)$, the completion $A_{p}$ of the normed algebra $A / \operatorname{ker} p$ with norm $\left\|x_{p}\right\|_{p}=p(x), x_{p}=x+\operatorname{ker} p$, is a uniform Banach algebra, hence commutative; and, as in [6, Theorem 5.1], $A$ is an inverse limit of uniform Banach algebras $A_{p}$ viz., $A=\varliminf_{p \in S\langle A\rangle} A_{p}$. Such an $A$ is a locally $m$-convex algebra [6], i.e., a topological algebra whose topology is determined by a family of algebra seminorms. The bounded part of $A$ is $b(A)=\left\{x \in A \mid \sup _{p \in S\langle A\rangle} p(x)<\infty\right\}$, a subalgebra of $A$ that is easily seen to be a uniform Banach algebra with norm $\|x\|_{\infty}=\sup _{p \in S\langle A\rangle} p(x)$ continuously embedded in $A$. A topological algebra $A$ is a $Q$-algebra [8, Chapter 1] if the set $A^{-1}$ of invertible elements forms an open set.

\section{ProOFs}

Proof of Theorem 1. (i) Let $|\cdot|$ be a linear norm with square property on a commutative algebra $A$. By commutativity, for all $x, y$ in $A, 4 x y=$ $(x+y)^{2}-(x-y)^{2}$, so that $2|x y| \leq(|x|+|y|)^{2}$. This gives $|x y| \leq 2$ for $|x| \leq 1,|y| \leq 1$ and so, by bilinearity, $|x y| \leq 2|x||y|$ for all $x, y$. For $n \in \mathbb{N}$ this gives $\left|x^{2^{n}} y^{2^{n}}\right| \leq 2\left|x^{2^{n}}\right|\left|y^{2^{n}}\right|,|x y|^{2^{n}} \leq 2(|x||y|)^{2^{n}},|x y| \leq 2^{1 / 2^{n}}|x||y|$. Since $n$ is arbitrary, $|x y| \leq|x||y|$.

(ii) In a normed algebra $(B, p)$, the limit $r^{\prime}(x)=\lim _{n} p\left(x^{n}\right)^{1 / n}$ exists [1, Proposition 2.8] and $r^{\prime}(x) \leq r(x)$, the spectral radius [1, Theorem 5.7]. Also, among normed algebras, $Q$-algebra is known to be characterized by the spectral radius formula $r^{\prime}(x)=r(x)$ [2, Proposition 15]. Using this in $(A,|\cdot|)$ together with the uniform property of $|\cdot|$ and $\|\cdot\|$, we get $|x|=\lim _{n}\left|x^{n}\right|^{1 / n}=r^{\prime}(x)=$ $r(x)=\|x\|$ for all $x$.

Proof of Corollary. Let $K$ be the Gelfand space of $A$, a compact Hausdorff space consisting of all nonzero multiplicative linear functionals on $A$. Let $x \in A \rightarrow \hat{x} \in C(K)$ be the Gelfand transform. Let $K_{1}=\{\phi \in K \mid \phi$ is $|\cdot|$-continuous $\}$. Then $K_{1} \subset K$. We claim that $\bar{K}_{1}=K$. If not, there exists an open set $G$ in $K$ such that $\bar{G} \subset K \backslash K_{1}$. The regularity of $(A,\|\cdot\|)$ [5, Corollary 7.3.4] implies that there exists an $x \in A$ such that $\hat{x}(\phi)=1$ for all $\phi \in K_{1}, \hat{x}(\phi)=0$ for all $\phi \in \bar{G}$. Let $\widetilde{A}$ be the completion of $(A,|\cdot|)$. Then 
$x$ is invertible in $\widetilde{A}$, because if not then there exists a multiplicative functional on $A$ whose restriction $\phi_{0} \in K_{1}$ satisfies $\hat{x}\left(\phi_{0}\right)=0$, a contradiction. Again by regularity of $(A,\|\cdot\|)$, choose a $y \in A$ such that $y \neq 0$, supp $\hat{y} \subset \bar{G}$. Then $(y x)^{\wedge}=\hat{y} \hat{x}=0$ on $K, y x \in \operatorname{Rad}(A)$, hence $y x=0$. Multiplying by $x^{-1}$, $y=0$, a contradiction. Thus $\bar{K}_{1}=K$. Now for all $x$ in $A$,

$$
\begin{aligned}
|x| & \geq \lim _{n}\left|x^{n}\right|^{1 / n}=r_{\vec{A}}(x) \\
& =\sup _{\phi \in K_{1}}|\hat{x}(\phi)|=\sup _{\phi \in K}|\hat{x}(\phi)|=r_{A}(x)=\|x\|
\end{aligned}
$$

establishing the first assertion. Thereby, additionally, if $|\cdot|$ is a uniform norm then Theorem 1(ii) applies giving $\|\cdot\|=|\cdot|$.

Proof of Theorem 2. In a complete uniform topological algebra $A \quad b(A)=\{x \in$ $A \mid S p_{A}(x)$ is bounded for all $\left.x\right\}$. Indeed, $A$ being complete and locally $m$ convex, $[8$, Theorem 12.8] implies that for each $x$

$$
S p_{A}(x)=\bigcup\left\{S p_{A_{p}}\left(x_{p}\right) \mid p \in S(A)\right\}
$$

and

$$
r(x)=\sup _{p \in S\langle A\rangle} \limsup _{n \rightarrow \infty} p\left(x^{n}\right)^{1 / n}=\sup _{p \in S\langle A\rangle} p(x)
$$

in view of $p\left(x^{2}\right)=p(x)^{2}$.

Further, by [6, Appendix E], in a $Q$-algebra, each element has bounded spectrum. Thus $b(A)=A$. Now since $A^{-1}$ is open, there exists a $p \in S(A)$ and $\varepsilon>0$ such that $S_{\varepsilon}=\{x \in A \mid p(1-x)<\varepsilon\} \subset A^{-1}$. We show that $\operatorname{ker} p=(0)$. Let $x \in \operatorname{ker} p, x \neq 0$. There is a $q \in S(A)$ such that $x_{q}=x+\operatorname{ker} q \neq 0$ in $A_{q}$. Since $q\left(x^{2}\right)=q(x)^{2}$, it follows that $x_{q}^{2} \neq 0$. Thus, for some $\lambda \neq 0$, $\lambda \in S p_{A_{q}}\left(x_{q}^{2}\right) \subset S p_{A}\left(x^{2}\right)$, and so $1-\lambda^{-1} x^{2}$ is not invertible in $A$. But $p\left(1-\left(1-\lambda^{-1} x^{2}\right)\right)=\lambda^{-1} p\left(x^{2}\right)=\lambda^{-1} p(x)^{2}=0$ giving $\left(1-\lambda^{-1} x^{2}\right) \in A^{-1}$, a contradiction. Thus ker $p=(0)$; and $p$ is a uniform norm on the uniform Banach algebra $\left(b(A),\|\cdot\|_{\infty}\right)$. Also $S_{\varepsilon} \subset A^{-1}$ shows that $(b(A), p)$ is a $Q$ algebra. Part (ii) of Theorem 1 shows that $p(\cdot)=\|\cdot\|_{\infty}$ on $A$ showing that the topology of $A$ is determined by $\|\cdot\|_{\infty}$.

\section{CONCLUDING REMARKS AND EXAMPLES}

3.1. Uniform Fréchet algebras (uF-algebras) have been extensively investigated by Kramm (see references in [6] and the forthcoming book by Goldmann [3]) in view of recapturing holomorphy through a functional analytic approach. They also have a bearing with the famous Michael problem [6]: Whether every multiplicative linear functional on a Fréchet $m$-convex algebra is necessarily continuous. Schottenloher [7] has discussed a class of nuclear DFN-spaces $E$ such that the Michael problem has a solution in general iff it has a solution for the uF-algebras $\theta(E)$ (in this case, nuclear and having Schauder basis) consisting of all holomorphic functions on $E$ with compact open topology. Let $A$ be a non-Banach uF-algebra. Then $A^{-1}$ is a $G_{\delta}$-set by [8, Theorem 1.6] that is not open by Theorem 2 , hence by [9, Corollary 3], not every element of $A$ has bounded spectrum. Thus by [9, Corollary 1], $A$ possesses a dense maximal ideal of infinite codimension (the content of Michael problem is [8, p. 87]: Whether every dense maximal ideal is of infinite codimension); and [ 9 , 
Theorem 1] implies that $A^{-1}$ fails to be open in any complete locally $m$-convex topology on $A$. Theorem 2 also implies, in view of [8, Theorem 12.21], that $A$ has the extension property that the convergence of a power series $\sum a_{n} x^{n}\left(a_{n}\right.$ scalars) for all $x$ in an open subset of $A$ implies its convergence for all $x$ in A.

3.2. The assumptions regarding openness of $A^{-1}$ in Theorem 1 and regularity in its corollary cannot be omitted. Let $D=\{z \in \mathbb{C}|| z \mid \leq 1\}$, let $A(D)$ be the disc algebra consisting of functions continuous on $D$ and holomorphic in int $D$. It is a uniform Banach algebra with norm $\|f\|=\sup \{|f(z)||| z \mid=1\}$. For any $a, 0<a<1,|f|=\sup \{|f(z)||| z \mid=a\}$ defines a uniform algebra on $A(D)$. By [5, p. 167], $\left(A(D),\|\cdot\|_{\infty}\right)$ is not regular and $A(D)^{-1}$ does not form an open set in $|\cdot|$, otherwise $\|f\|=r(f)=|f|(f \in A(D))$ by [2, Proposition 15].

3.3. Theorem 1(ii) is related to a well-known result that if $A$ is a subalgebra of $C(X)$, for a compact Hausdorff $X$, that is a Banach algebra with some norm $|\cdot|$, then supnorm $\|\cdot\|_{\infty} \leq|\cdot|$. Even if $\left(A,\|\cdot\|_{\infty}\right)$ is a $Q$-algebra, it is no longer true that $\|\cdot\|_{\infty}=|\cdot|$. Take the Banach algebra $C^{1}[0,1]$ consisting of $C^{1}$-functions on $[0,1]$ with norm $|f|=\|f\|_{\infty}+\left\|f^{\prime}\right\|_{\infty}$. It is easily seen that $\left(C^{1}[0,1],\|\cdot\|_{\infty}\right)$ is a $Q$-algebra. However, if $(A,|\cdot|)$ is a uniform algebra such that either (a) it is regular or (b) $(A,\|\cdot\|)$ is a $Q$-algebra, then $\|\cdot\|=|\cdot|$.

3.4. In passing, we inquire whether the uniform algebra is determined locally, i.e., let $(A,\|\cdot\|)$ be a commutative Banach algebra such that for each $x$ in $A$, the closed subalgebra $C(x)$ generated by 1 and $x$ admits a norm $p_{x}$ such that $\left(C(x), p_{x}\right)$ is a uniform Banach algebra. Is $A$ a uniform Banach algebra under an equivalent norm? By uniqueness of topology on semisimple Banach algebra [1, Theorem 25.9], $p_{x}$ determines the relative $\|\cdot\|$-topology on $C(x)$.

3.5. The assumption regarding the uniform property of the topology in Theorem 2 is crucial. Consider the algebra $A=C^{\infty}[0,1]$ of all $C^{\infty}$-functions on $[0,1]$ with topology $\tau$ defined by the algebra seminorms

$$
p_{n}(f)=\sup _{0 \leq t \leq 1}\left[\sum_{k=0}^{n}\left(\left|f^{(k)}(t)\right| / k !\right)\right] \text {. }
$$

Then $(A, \tau)$ is a Fréchet $m$-convex algebra that is a $Q$-algebra [6, Appendix E] and $\tau$ is not Banachizable. In fact, $A$ is non-Banachizable with any norm, since a semisimple commutative Banach algebra is known not to admit a nonzero derivation [1, Theorem 18.21]. The algebra $(A, \tau)$ is not a uniform topological algebra, either by Theorem 2 , or by noting that otherwise $b(A)=A$ has to be a uniform algebra.

3.6. Let $U$ be the open unit disc. Consider the complete uniform topological algebra $E$ consisting of all entire functions and $H(U)$ consisting of all holomorphic functions on $U$, both with compact open topology. Then $b(E)=\mathbb{C}$ by Liouville's theorem; and $b(H(U))=H^{\infty}(U)$, the uniform Banach algebra of all bounded holomorphic functions on $U$, which is dense in $H(U)$. This suggests the following question. Given a complete uniform topological algebra $A$, is it true that either $b(A)$ is finite-dimensional or $b(A)$ is dense in $A$ ? 
3.7. The following problem (suggested by the referee) is related with Theorem 1. Let $A$ be an algebra not necessarily commutative. Let $|\cdot|$ be a (semi)norm on $A$ satisfying $\left|x^{2}\right|=|x|^{2}$ for all $x$. Does it follow that $|x y| \leq|x||y|$ for all $x, y$ ?

\section{ACKNOWLEDGMENT}

The authors are thankful to Professor M. H. Vasavada for very fruitful discussions. Thanks are also due to the referee for several suggestions regarding terminology and simplification in the proof of Theorem 1(i).

\section{REFERENCES}

1. F. F. Bonsall and J. Duncan, Complete normed algebras, Springer-Verlag, Berlin, Heidelberg, and New York, 1973.

2. S. J. Bhatt, On spectra and numerical ranges in locally m-convex algebras, Indian J. Pure Appl. Math. 14 (1983), 596-603.

3. H. Goldmann, Uniform Fréchet algebras, North-Holland, Amsterdam, 1990.

4. B. Kramm, A duality theorem for nuclear function algebras, Aspects of Mathematics and its Applications (J. A. Barroso, ed.), Elsevier, Amsterdam, 1986, pp. 495-532.

5. R. Larsen, Banach algebras, Marcel-Dekker, New York, 1973.

6. E. Michael, Locally multiplicatively convex topological algebras, Mem. Amer. Math. Soc., vol. 11, Amer. Math. Soc., Providence, RI, 1952.

7. M. Schottenloher, Michael problem and the algebras of holomorphic functions, Ark. Mat. 37 (1981), 241-247.

8. W. Żelazko, Selected topics in topological algebras, Univ. Lecture Notes in Math., vol. 31, Aarhus, 1971.

9. __ On maximal ideals in commutative m-convex algebras, Studia Math. 58 (1976), 291298.

Department of Mathematics, Sardar Patel University, Vallabh Vidynagar-388 120 , GuJARAT, INDIA 